\title{
Experimental Evidence for Vibrational Entropy as Driving Parameter of Flexibility in the Metal-Organic Framework ZIF-4(Zn)
}

DOI:

10.1021/acs.chemmater.9b01908

\section{Document Version}

Accepted author manuscript

Link to publication record in Manchester Research Explorer

Citation for published version (APA):

Butler, K. T., Vervoorts, P., Ehrenreich, M. G., Armstrong, J., Skelton, J. M., \& Kieslich, G. (2019). Experimental Evidence for Vibrational Entropy as Driving Parameter of Flexibility in the Metal-Organic Framework ZIF-4(Zn). Chemistry of Materials, 31(20), 8366-8372. https://doi.org/10.1021/acs.chemmater.9b01908

Published in:

Chemistry of Materials

\section{Citing this paper}

Please note that where the full-text provided on Manchester Research Explorer is the Author Accepted Manuscript or Proof version this may differ from the final Published version. If citing, it is advised that you check and use the publisher's definitive version.

\section{General rights}

Copyright and moral rights for the publications made accessible in the Research Explorer are retained by the authors and/or other copyright owners and it is a condition of accessing publications that users recognise and abide by the legal requirements associated with these rights.

\section{Takedown policy}

If you believe that this document breaches copyright please refer to the University of Manchester's Takedown Procedures [http://man.ac.uk/04Y6Bo] or contact uml.scholarlycommunications@manchester.ac.uk providing relevant details, so we can investigate your claim.

\section{OPEN ACCESS}




\title{
Experimental Evidence for Vibrational Entropy as Driving Parameter of Flexibility in the Metal-Organic Framework ZIF-4(Zn)
}

\author{
Keith T. Butler, ${ }^{* a}$ Pia Vervoorts, ${ }^{b}$ Michael G. Ehrenreich, ${ }^{b}$ Jeff Armstrong, ${ }^{a}$ Jonathan \\ M. Skelton, ${ }^{d}$ Gregor Kieslich ${ }^{* b}$
}

aISIS Facility, Rutherford Appleton Laboratory, Harwell Oxford, Didcot, Oxfordshire OX11 0QX, UK.

bDepartment of Chemistry, Technical University of Munich, Lichtenbergstraße 4, 85748 Garching, Germany.

dSchool of Chemistry, University of Manchester, Oxford Road, Manchester M13 9PL, UK

*corresponding authors: Keith T. Butler, keith.butler@stfcac.uk

Gregor. Kieslich, Gregor.Kieslich@tum.de

\begin{abstract}
Large structural flexibility in some metal-organic frameworks (MOFs) is not only fascinating from a fundamental point of view but offers exciting opportunities in the areas of gas sensing, gas separation and mechanocalorics. After intense interest of the MOF and materials community in these materials, scientists are starting to understand the details and principles that govern the thermodynamics of them from theoretical models, however experimental confirmation of possible models is still outstanding. We here use temperature dependent inelastic neutron scattering to track the changes of lattice dynamics across the closed-pore to open-pore phase transition in ZIF-4(Zn). Our experiments are complemented by lattice dynamics calculations, allowing us to identify the important structural features that govern the free energy landscape in ZIF-4(Zn). We find that rigid unit modes dominate the low energy lattice dynamics, suggesting the implementation of rigid unit modes in the search for guiding principles for the synthesis of flexible MOFs.
\end{abstract}

\section{Introduction}

Metal-organic frameworks (MOFs) are a fascinating class of materials, ${ }^{1,2}$ combining the nearly unlimited parameter space of organic chemistry with the large library of inorganic metal nodes. The modular building principle of MOFs as embodied in the reticular chemistry approach allows for designing open pore frameworks with distinct structures and chemical functionalities, ${ }^{3}$ making MOFs attractive for researchers from various areas across materials sciences. $A$ subclass of MOFs, oftentimes referred to as soft porous crystals (SPCs) or flexible MOFs, ${ }^{4-9}$ exhibit structural flexibility with large volume changes when triggered by an external stimulus ${ }^{10}$ such as temperature,${ }^{11}$ pressure,${ }^{12}$ light ${ }^{13}$ or gas and guest adsorption. ${ }^{14}$ Structural flexibility in SPCs is not only fascinating from a fundamental point of view but also offers striking opportunities in the areas of gas separation ${ }^{15,16}$ and sensing,,$^{17}$ mechanocalorics ${ }^{18}$ and their application as mechanical dampers and shock absorbers. ${ }^{19}$ Prototypical examples of SPCs that exhibit a wine-rack type structure motif are MIL-53 (MIL = Matériaux de I'Institut Lavoisier; $\mathrm{M}(\mathrm{OH})(\mathrm{bdc}) ; \mathrm{M}^{3+}=\mathrm{Al}^{3+}, \mathrm{Fe}^{3+}, \mathrm{Cr}^{3+}$ etc., bdc $^{2-}=1,4-$ benzenedicarboxylate) $){ }^{20-22}$ DUT-8 (DUT = Dresden University of Technology, $\mathrm{M}^{2+}=\mathrm{Ni}^{2+}, \mathrm{Zn}^{2+}, \mathrm{Co}^{2+}, \mathrm{M}_{2}(2,6-\mathrm{ndc})_{2} \mathrm{dabco}$, $\mathrm{ndc}^{2-}=2,6-$ naphtalenedicarboxylate, dabco $=1,4$-diazabicyclo[2.2.2]octane $)^{23,24}$ and the material class of pillared-layered MOFs with the general formula $\mathrm{M}_{2}(\mathrm{fu}-\mathrm{bdc})_{2}$ (dabco) (fuMOFs, $\mathrm{M}=\mathrm{Cu}^{2+}$ or $\mathrm{Zn}^{2+}$, fu-bdc ${ }^{2-}=2,5$-difunctionalized-1,4-benzendicarboxylate $)^{25,26}$ which exhibit structural flexibility 
as response to gas sorption, temperature variation and/or hydrostatic pressure..$^{19,27}$ The topologically related material [ $\mathrm{Zn}_{2}\left(4,4\right.$ '-biphenyldicarboxylate) ${ }_{2}\left(4,4^{\prime}\right.$-bis(4-pyridyl)biphenyl)] combines many different aspects of structural flexibility in one single material, ${ }^{28}$ e.g. a breathing motion, structural isomerism and a shape memory effect. Other intriguing SPCs are Co(bdp) and $\mathrm{Fe}(\mathrm{bdp})$ (bdp ${ }^{2-}=1,4$-benzendipyrazolate), ${ }^{29}$ in which a high $\mathrm{CH}_{4}$-storage capacity is combined with an effective internal heat management through lattice expansion/contraction work upon $\mathrm{CH}_{4}$ adsorption/desorption. Arguably the most complex example with respect to structural deformation and thermodynamic parameters presently is DUT- $49,{ }^{30}$ showing a very unusual structural evolution as function of $\mathrm{CH}_{4}$ or $\mathrm{C}_{4} \mathrm{H}_{10}$ pressure.

Over the past decade, there has been an intense interest in developing a thorough understanding of SPCs, with the goal being the formulation of design guidelines for the targeted synthesis of SPCs. Topological, ${ }^{31}$ symmetry-related ${ }^{32}$ and thermodynamic aspects $^{33,34}$ have been identified as key factors for stimulus responsive behaviour to occur, leading to numerous chemical and structural requirements that must be met at the same time. The largest progress in this context has undoubtedly come from theory, where various computational methods have been applied to predict and study flexibility and thermodynamic parameters within SPCs, constantly challenging available methods and computational expenses. ${ }^{10,18,35-37}$ When temperature is used as a trigger for structural flexibility in SPCs, the thermodynamic balance might be simplified to a balance between dispersion interactions and vibrational entropy, i.e. $\Delta G=\Delta H_{\text {Dispersion }}-\mathrm{T} \Delta S_{\text {Vibrational, }}{ }^{36,38,39}$ having in mind that contributions from configurational entropy can arise in certain materials. ${ }^{26}$ Despite the simplification in this particular scenario, the study and the manipulation of this balance still challenges both experimentalists and computational scientists with currently only qualitative guidelines existing. ${ }^{39}$ Several groups have investigated the free energy landscape of SPCs computationally, all confirming the crucial role of vibrational entropy and dispersion interactions. For instance, early on Walker et al. have noted the importance of dispersion interactions in the flexibility of MIL-53(Al). ${ }^{34}$ Likewise, E. Cockayne has recently applied the quasi-harmonic approximation for explicitly studying the Helmholtz and Gibbs free energy as a function of temperature of MIL-53(Cr). ${ }^{33}$ In accordance with related studies and recently reported results, ${ }^{34,36}$ the treatment of Van der Waals interactions was again emphasized. Therefore, theory has shown to be highly successful in investigating the balance between $\Delta S_{\text {Vibrational }}$ and $\Delta H_{\text {Dispersion, }}{ }^{36,40}$ providing insight into this delicate balance that must be met for structural flexibility to occur. Focusing on the origin of vibrational entropy, the presence of low energy lattice modes that can be populated at elevated temperature have shown to be of utmost importance..$^{41-43}$ In turn, it is clear that the explicit study of lattice dynamics experimentally and computationally - can provide invaluable understanding into the 


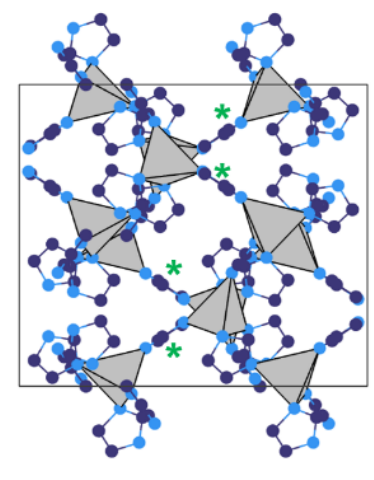

ZIF-4(Zn)-cp

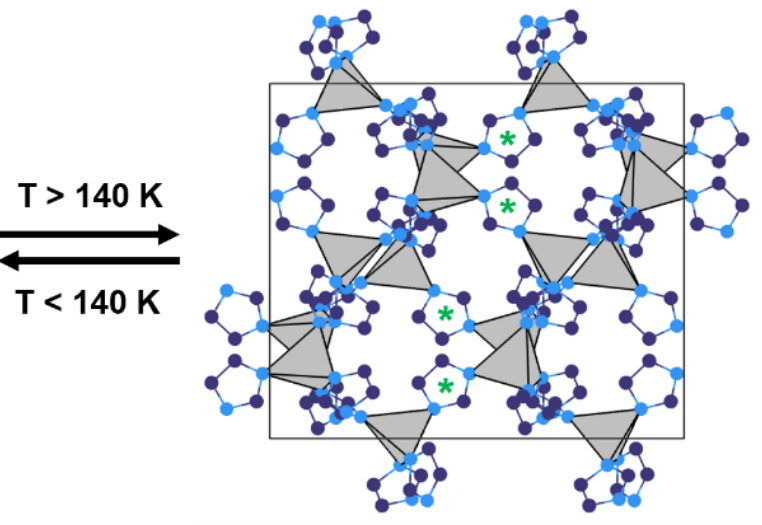

ZIF-4(Zn)-op

Figure 1. Schematic of the low temperature structure of ZIF-4(Zn)-cp and the high temperature structure ZIF-4(Zn)-op, looking down the $b$-axis in both phases. The volume contraction of approximately $24 \%$ that comes with the phase transition is related to a rotation of the imidazolate linkers. Four of such rotations are highlighted by green stars (colour code: dark blue: nitrogen, light blue: carbon and grey tetrahedrons: $\mathrm{ZnN}_{4}$ units).

thermodynamics and how to experimentally manipulate these in SPCs. Despite the abundance of theoretical models, there is a surprising lack of experimental insight into the lattice dynamics of SPCs. When combined with theory, experimental studies can provide an additional perspective and understanding into the free energy landscape of SPCs.

Here we use temperature dependent inelastic neutron scattering (INS) to experimentally study the change of low energy lattice dynamics in the flexible SPC ZIF-4(Zn) as a function of temperature for the first time. We use our experimental results as a benchmark for $a b$ initio lattice dynamic calculations within the quasi-harmonic approximation, explicitly studying the impact of vibrational entropy as a function of temperature. Recently, Tan et al. applied inelastic neutron scattering and terahertz spectroscopy at low temperatures to MOFs, ${ }^{44,45}$ showing that INS is a valuable tool that allows for accessing lattice dynamics and shear deformations in a series of imidazolate frameworks. We expand on their work, experimentally following the changes of lattice dynamics as a function of temperature and shedding light on the thermodynamic origin of temperature-driven flexibility in ZIF-4(Zn) (zeolitic imidazolate framework, $\mathrm{Zn}(\mathrm{im})_{2}$ with $\mathrm{im}^{-}=$imidazolate) across the temperature-driven phase transition. ${ }^{46,47}$ $\mathrm{ZIF}-4(\mathrm{Zn})$ is a thermo, ${ }^{48}$ pressure ${ }^{49,50}$ and guest-responsive SPC with a volume change of up to $24 \%{ }^{48}$ when going from the low-temperature/high-pressure closed-pore phase (ZIF-4(Zn)$\mathbf{c p )}$ to the high-temperature/low-pressure open-pore phase (ZIF-4(Zn)-op), see Figure $\mathbf{1}$ for an illustration of the structures. Importantly, the phase transition occurs at approximately $T=$ $140 \mathrm{~K}$, making the phase transition accessible by INS..$^{51,52}$ In fact, the relatively larger number of hydrogen atoms and nature of the closed-pore to open-pore phase transition in ZIF-4(Zn), which is related to a rotation of imidazolate linkers makes this type of phase transition ideal for such a study. We identify vibrational entropy as an important factor that drives the ZIF-4(Zn)- 
cp to ZIF-4(Zn)-op phase transition at elevated temperatures, discovering a pronounced effect of collective low energy lattice modes that are best described as rigid unit modes (RUMs). This understanding of the microscopic details of the thermodynamics of the phase transition allows us to propose potential future approaches for developing design principles of SPCs.

\section{Results and discussion}

ZIF-4(Zn) powder was synthesised following a standard procedure from the literature, see Ref. 47,48 and supporting information (SI) for details. Prior to the INS experiments, ZIF-4(Zn) was thoroughly activated by solvent exchange and subsequent solvent removal at $423 \mathrm{~K}$ under dynamic vacuum. Powder X-ray diffraction was used to confirm phase purity and complete activation of ZIF-4(Zn) prior to INS measurements, see SI for details. For INS experiments, approximately $2.5 \mathrm{~g}$ of activated $\mathrm{ZIF}-4(\mathrm{Zn})$ were filled into an $\mathrm{Al}$ can and sealed under $\mathrm{Ar}$ atmosphere. INS experiments as a function of temperature between $10 \mathrm{~K}$ and $180 \mathrm{~K}$ of ZIF$4(\mathrm{Zn})$ were recorded at the inverted-geometry neutron spectrometer TOSCA located at the ISIS Pulsed Neutron \& Muon Source, Rutherford Appleton laboratory (see SI for details). Ab initio DFT based lattice dynamic calculations have been performed within the quasi-harmonic approximation using the VASP code and the PHONOPY package, ${ }^{53}$ see SI for details. We would like to emphasize that the quasi-harmonic approximation only includes lattice expansion as additional parameter and does not explicitly account for anharmonic contributions. Both, ZIF-4(Zn)-cp and ZIF-4(Zn)-op have been considered in the calculations and the temperature dependent INS spectra were simulated using the ABINS package as integrated in the lasted version of the Mantid program. ${ }^{54,55}$ The experimental and simulated INS spectra for the low temperature ZIF-4(Zn)-cp and high temperature ZIF-4(Zn)-op phases are shown in Figure 2a -c. The measured spectrum at $T=10 \mathrm{~K}$ is in good agreement with the simulated INS spectrum of ZIF-4(Zn)-cp, see Figure 2c, and with previous measurements from Tan et al., see SI for details. In our case, we observe an excellent agreement between theory and experiment for modes with frequencies higher $500 \mathrm{~cm}^{-1}$. For the low energy region, the agreement is qualitative with largest difference being the shoulder at around $80 \mathrm{~cm}^{-1}$ in our simulations of ZIF-4(Zn)-cp (Figure 2c), which in the experiment seems to be shifted to slightly higher frequencies $80-90 \mathrm{~cm}^{-1}$ (Figure $\mathbf{2 b}$, light blue curve). In the simulated spectra of ZIF-4(Zn)op, this mode gains in intensity (Figure 2a). The experiment similarly shows changes of intensities in the frequency region $80-100 \mathrm{~cm}^{-1}$ but not as pronounced as in the simulations. It should be noted that a full quantitative agreement between INS and lattice dynamic calculations still challenges todays computational methodologies, with only a few examples known where quantitative agreement between experiment and theory has been obtained. ${ }^{42,44,51,56,57}$ Looking at the low frequency region with frequencies $<50 \mathrm{~cm}^{-1}$ subtle but significant differences are observed as a function of temperature (Figure $\mathbf{2 b}$, grey region), which are reproduced in the simulated INS spectrum of ZIF-4(Zn)-op. It has previously been 


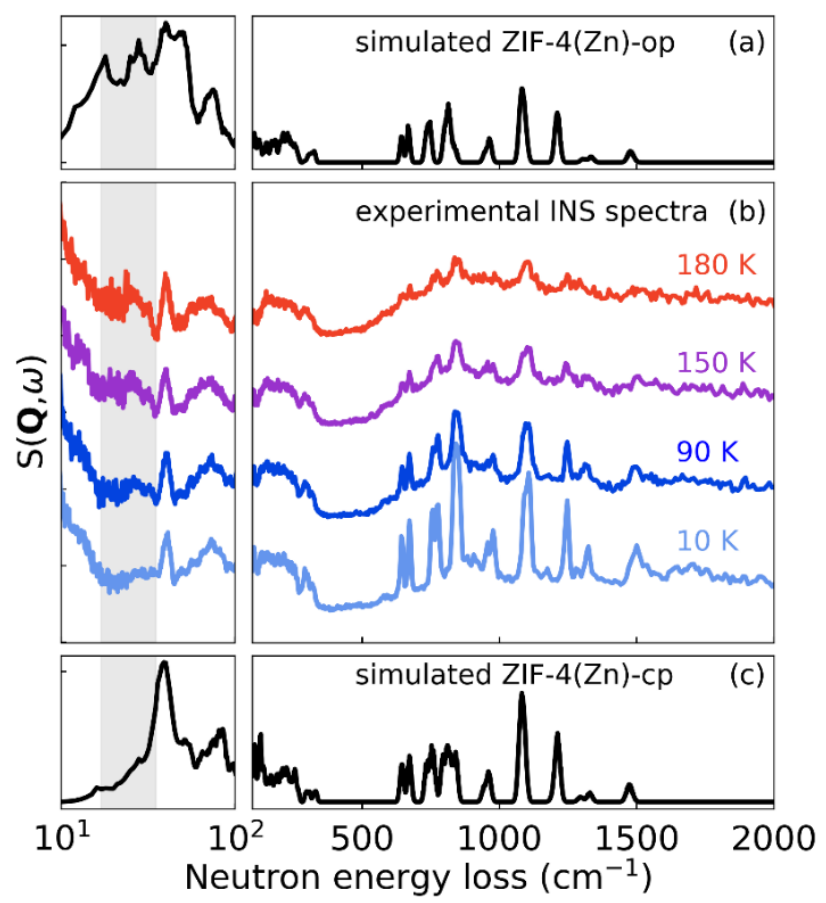

Figure 2. Experimental and simulated inelastic neutron spectra of ZIF-4(Zn)-cp and ZIF4(Zn)-cp. The simulated INS spectra of ZIF-4(Zn)-op is shown in (a), and that of ZIF-4(Zn)$\mathbf{c p}$ in (c). The experimental INS spectra are shown in panel (b). A Boltzmann correction factor, $\exp \left(-E / k_{b} T\right)$, has been applied to all experimental INS spectra.

shown, that such small differences are within the resolution of INS, particularly at these low energies where typically peak broadening coming from phonon dispersion limits higher resolutions. ${ }^{58}$ These low energy features, sometimes referred to as external modes, correspond to collective phonon modes which are a fingerprint of the lattice dynamics of the respective thermodynamic state of $\mathrm{ZIF}-4(\mathrm{Zn})$ and therefore confirm the change of lattice dynamics when going from ZIF-4(Zn)-cp to ZIF-4(Zn)-op as a function of temperature. The general signatures of the high energy regime $\left(\omega>500 \mathrm{~cm}^{-1}\right)$, which relate to internal molecular vibrations remain mainly unchanged, confirming that the phase transition is related to a linker rotation rather than a linker deformation. ${ }^{48}$ Only peak-broadening is observed due to increasing contributions from the Debye-Waller factor in $S(\mathbf{Q}, \omega){ }^{52}$ Given the agreement between computational and experimental INS spectrum, we can conclude that the applied lattice dynamic calculations are sufficiently reproducing the lattice dynamics, giving us confidence in the general applicability of the quasi-harmonic approximation for ZIF-4(Zn) for describing the lattice dynamics in this system.

In order to investigate the impact of lattice dynamics on the free energy landscape and in turn on the structural response of ZIF-4(Zn), the temperature dependent entropy and Gibbs energy of the phases as function of temperature were calculated according to (1):

$$
G(T, P)=\min _{V}\left[U(V)+F_{p h}(V, T)+p V\right],
$$




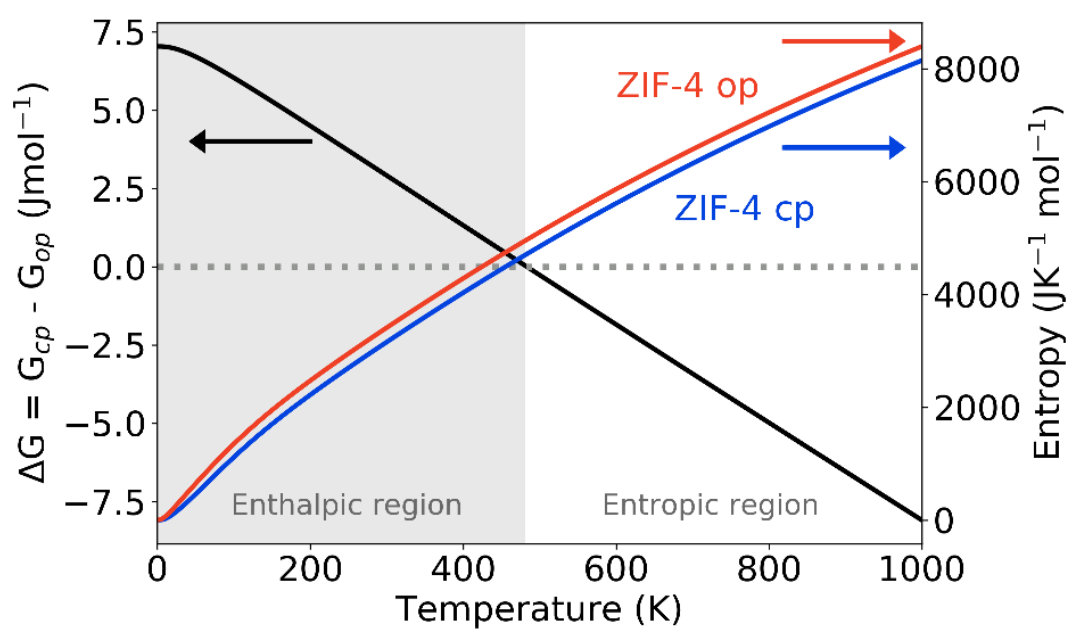

Figure 3. Calculated Gibbs free enthalpy, $\Delta G(T)=G_{\mathrm{OP}}-G_{\mathrm{CP}}$ (black), and entropies $S_{\mathrm{OP}}$ (red) and $S_{\mathrm{CP}}$ (blue) as a function of temperature for ZIF-4-cp and ZIF-4-op. The phase transition is driven by the larger entropy of the op phase and hence by the term $T \Delta S$. The simulated phase transition temperature is $T_{\text {cp-op }}=485 \mathrm{~K}$.

with $G$ the Gibbs free energy equal to the minimised value of the sum in brackets with respect to the volume $V$; $U$ is the enthalpy, $F_{\mathrm{ph}}$ is the Helmholtz free energy as obtained in the harmonic approximation and $p V$ is the pressure volume contribution. The obtained Gibbs free energy difference, $\Delta G(T)=\mathrm{G}_{\mathrm{CP}}(T)-\mathrm{G}_{\mathrm{OP}}(T)$, is shown in Figure 3, plotted as a function of temperature. $T=485 \mathrm{~K}, \Delta G(T)$ becomes negative, making the ZIF-4(Zn)-op phase the thermodynamic stable modification. The change is driven by the large gain of vibrational entropy of the ZIF4(Zn)-op modification, $T \Delta S(T)=T\left(\mathrm{~S}_{\mathrm{OP}}(T)-\mathrm{S}_{\mathrm{CP}}(T)\right)$, which outweighs differences of $\Delta H$ at high temperatures (see coloured curves in Figure 3). Looking at the origin of vibrational entropy in more detail, it is fruitful to look at the entropy expression of the entropy in the quasi harmonic approximation:

$$
S(V)=-\frac{1}{N} \sum_{k, i} k_{B} \ln \left[1-\exp \left(-\frac{h v_{k, i}(V)}{k_{B} T}\right)\right]+\frac{1}{N} \sum_{k, i} k_{B} \frac{h v_{k, i}(V)}{k_{B} T}\left[\exp \left(\frac{h v_{k, i}(V)}{k_{B} T}\right)-1\right]^{-1}
$$

Here, $\mathrm{N}$ is the number of modes (i) times the number of q-points $(\mathrm{k}), \mathrm{h}$ is Plank's constant, $k_{\mathrm{B}}$ is Boltzmann's constant $v$ are the frequencies, $T$ is the temperature and $V$ is the volume. In both terms of this expression there is an inverse exponential dependence of the entropy on the frequency of the phonon mode. This inverse exponential relationship is important since it means that over all reasonable temperature ranges the low frequency vibrations dominate the vibrational entropy term.

The difference between the experimentally observed $(T=140 \mathrm{~K})$ and calculated $(T=485 \mathrm{~K})$ transition temperatures again emphasises the subtle balance of entropy and dispersion that governs flexibility, and, at the same time underlines the accuracy that is required to computationally reproduce experimental observations. In our calculations, the ZIF-4(Zn)-op 
phase has imaginary phonon frequencies, which can be expected for a high temperature phase. Following the approach by Cockayne ${ }^{33}$ we followed along these modes in the cell used for phonon calculations to arrive at the stable structure. In a next step, the phonons and thermodynamic properties of this new structure were calculated, which maintains the same Pbca symmetry. By applying this approach, we find that the calculated phase transition temperature decreases around $50 \mathrm{~K}$, see supporting information for details. Interestingly, when imaginary modes are treated as real by taking the absolute frequency for calculation of thermodynamic properties shows a similarly effect. While both approaches lead to an improvement, it also demonstrates that quantitatively there are several important factors that contribute to differences in the calculated and measured transition temperatures.

Importantly, it has been previously shown that in the absence of dispersion correction terms, standard density functional theory (DFT) fails entirely to reproduce the closed pore phase of ZIF-4. ${ }^{40}$ The dispersion forces are included in our simulations using the empirical D3 function; 59 however, it seems that the empirical D3 correction overcompensates in the current system, over-stabilising the ZIF-4(Zn)-cp phase and increasing the calculated transition temperature. Recent developments such as random phase approximation calculations of dispersion show great promise for obtaining accurate $a b$ initio dispersion terms to correct DFT ${ }^{36}$ Notably, the same study found a pronounced effect of the treatment of dispersion applied on the transition temperature of flexible MIL-53 systems, with different approaches shifting the transition by more than $450 \mathrm{~K}$. Additionally it has been noted that the weak divergence of terms for vibrational entropy as the frequency approaches zero can affect the relative energy of phases by up to $15 \% .{ }^{33}$ However, we like to emphasize that the goal of the study was not to establish or to benchmark computation, instead to understand the origin of large vibrational entropy and changes of lattice dynamics across the ZIF-4(Zn)-cp to ZIF-4(Zn)-op phase transition as a function of temperature which is discussed in the next paragraph.

To understand the origin of vibrational entropy differences between the phases of ZIF-4(Zn) more quantitatively, one must turn towards the explicit analysis of lattice modes. ${ }^{60}$ As we and others have shown previously, ${ }^{41,61}$ modes with frequencies up to $\sim 333 \mathrm{~cm}^{-1}$ (approx. $10 \mathrm{THz}$ ) account for the majority of vibrational entropy. With this in mind, the difference in frequency, $\Delta \omega=\omega_{i, o p}-\omega_{j, c p}$ is plotted in Figure 4, with $\omega_{i, o p}$ and $\omega_{i, c p}$ being the frequency of mode $i$ of the op and $\mathrm{cp}$ form of ZIF-4(Zn). For the plot, certain regions have been binned together to emphasize on the general trends. A negative value of $\Delta \omega$ implies that the frequency is lower in the op phase, and therefore related to a gain in vibrational entropy and vice versa. Additionally, the colour of the data points in Figure 4 indicates the fractional ligand/linker contribution to each mode. It can be observed that there is a manifold of low frequency modes that soften in ZIF-4(Zn)-op compared to ZIF-4(Zn)-cp, predominantly consisting of collective modes shared between linker and ligand with a slightly higher weighting towards the ligand. 


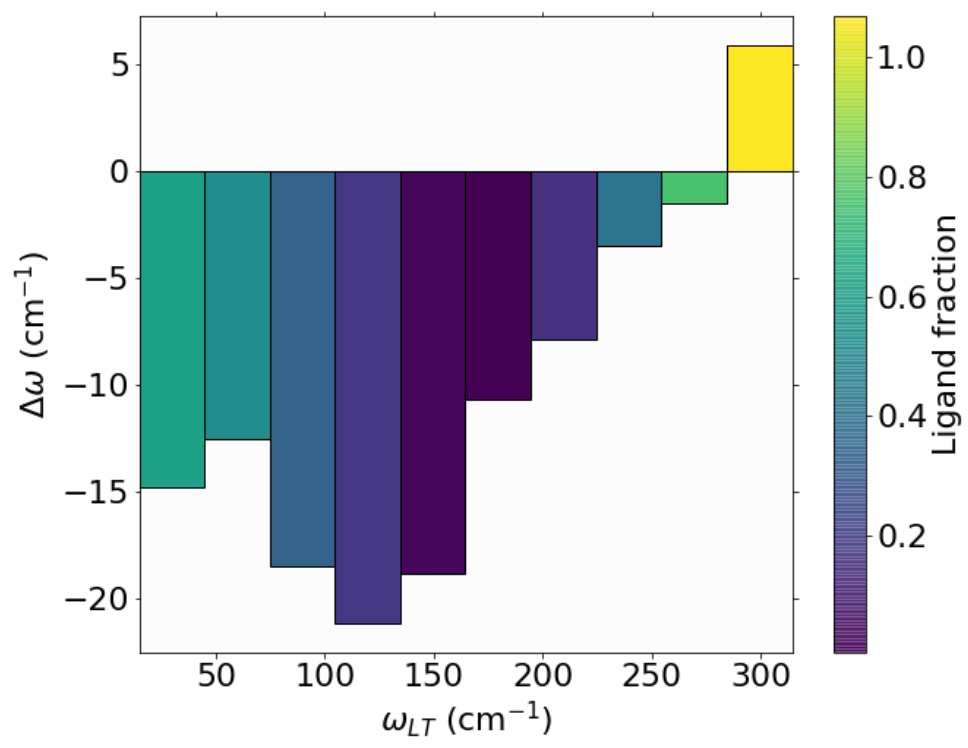

Figure 4. Difference of mode frequencies between the ZIF-4(Zn)-op and ZIF-4(Zn)-cp with a negative value indicating a softening of the mode and in turn an increase in vibrational entropy. The colour scheme of the data points reflects the fractional ligand/linker contribution to the respective mode.

Overall, the increase of vibrational entropy at higher temperature is therefore driven by a softening of low frequency modes which account for the higher vibrational entropy in ZIF-4(Zn)op. Interestingly, all modes up to $100 \mathrm{~cm}^{-1}$ that contribute to vibrational entropy are best described as rigid unit modes (RUMs), involving collective rotations of the $\mathrm{ZnN}_{4}$ tetrahedra with no variation of $\mathrm{Zn}-\mathrm{N}$ distances, see movies given in the SI. We like to emphasize that in these movies, $\mathrm{ZnN}_{4}$ tetrahedra are only shown if there is no variation of $\mathrm{Zn}-\mathrm{N}$ distances, applying a strict condition on the description of rigid unit modes. Within these rotating motions of the RUMs, neighbouring molecules move closer and further apart during the vibration. In the close pore structure, the distances between the molecules is smaller, so in the course of the vibration the electron density of neighbouring molecules overlaps to a greater extent. This increased overlap presumably results in greater Pauli repulsion, which then seems to stiffen the vibrational mode at the closed pore phase and hence the lower vibrational entropy. RUMs have previously been shown to be an elegant concept to describe displacive phase transitions and the phonon-based contributions to negative thermal expansion in various materials ${ }^{62-64}$. It will be therefore interesting to see if a similar formulism, or an adapted RUM concept that includes some sort of polyhedral variation ${ }^{65}$ can in the future account for collective structural deformations in SPCs. ${ }^{66}$

\section{Conclusion}

To our knowledge, this work presents the first example of a combined experimental and computational approach that focuses on experimentally following the changes of low energy 
lattice dynamics across the phase transition of SPCs. The results remind us of the significance of vibrational entropy in organic-inorganic frameworks in general and highlight its role in the structural flexibility of SPCs in particular. It is important to mention that entropy is a longestablished parameter in areas such as glasses, protein folding and alloys. ${ }^{67-70}$ Similarly, in related dense hybrid organic-inorganic materials, a delicate balance was recently found to exist between vibrational entropy, configurational entropy and hydrogen bonding, determining the phase evolution in such materials as a function of temperature. ${ }^{42}$ When looking at the potential types of weak interactions in SPCs (that in principal can be designed), and, in combination with the low physical as well as packing densities of these materials, it is surprising that only a limited number of studies have hitherto addressed thermodynamic particularly entropic influences in SPCs experimentally. ${ }^{11,71,72}$

Our results together with the recent discoveries in the field of SPCs bring us to the challenges that must be addressed when interested in the targeted design of SPCs. Inspired by the reticular chemistry approach, synthetic guidelines towards SPCs that make use of available topological databases seem the natural approach. The reticular chemistry approach as a successful rigid body approach intrinsically neglects topological, symmetry and thermodynamic parameters related to dynamic processes that render structural flexibility, ${ }^{39}$ and modifications must be applied. For instance, once a topology (from a database) has been tested for potential flexibility by applying classical counting rules or group theoretical considerations, ${ }^{31,32}$ the next step would be the combination of the given topology with available linkers and metal nodes. The existence of a flexibility of a topology-metal-node-linker combination could then be tested by searching for the occurrence of RUMs, as open-pore to closed-pore phase transitions are typically of displacive nature. RUMs are an appealingly simple concept and their existence can be predicted by using relatively cheap algorithms, for which different available codes exist. ${ }^{73}$ The arguably most challenging step is then the assessment of the free energy landscape of both phases, where a free energy landscape is required that exhibits distinct energy minima, but at the same time is shallow enough to facilitate responsivity of the material as function of an external stimulus. It currently seems as a good approximation that the vibrational entropy is larger in a less dense (potentially openpore) structure and is therefore predetermined. Therefore, dispersion interactions might be the tool of choice for experimentalists to access the free energy landscape synthetically. The manipulation of dispersion interactions has already proved to be a useful lever to move the entropy-dispersion balance towards the one or the other direction. For instance, side-chain functionalization of the bdc ${ }^{2-}$ linker within the materials series of $Z \mathrm{n}_{2}(\mathrm{fu}-\mathrm{bdc})_{2}(\mathrm{dabco})^{26}$ can render the materials thermoresponsive. ${ }^{26}$ Looking at ZIF-4(Zn), dispersion interactions thus could be accessed by linker functionalization, i.e. the partial incorporation of functionalized imidazolate linkers into the structures. It is important to mention that the above given outline 
neglects structural flexibility when linker deformation or metal-node deformation is involved;30,74 here however, ideas to extend the RUM model to so-called 'quasi-RUMs' have already been proposed, ${ }^{75,76}$ and potentially offers exciting opportunities for SPCs in the future. Therefore, it currently seems that the combination of topological control of vibrational entropy and the tunability of dispersion interactions offers great promise for the formulation of design guidelines of SPCs, with many challenging aspects out there that remain to be addressed by computation and experiment.

\section{Associated Content}

Experimental details and supporting information. Full experimental and computational details are provided in the supporting information including PXRD pattern before and after activation and a significance analysis of the imaginary modes. The supporting information is available free of charge on the ACS Publications website, see http://pubs.acs.org.

Author information. E-mail: Keith.butler@stfc.ac.uk and Gregor.Kieslich@tum.de

\section{Acknowledgements}

GK and MGE would like to thank the "Fonds der Chemischen Industrie" for support through the Liebig Fellowship scheme and financial support from the DFG priority program SPP1928 through affiliation as young investigator. PV acknowledges the financial support by the DFG (Deutsche Forschungsgemeinschaft) within the research unit 2433 "Switchable Metal-Organic Frameworks".

\section{References}

(1) Batten, S. R.; Champness, N. R.; Chen, X.-M.; Garcia-Martinez, J.; Kitagawa, S.; Öhrström, L.; O'Keeffe, M.; Paik Suh, M., Reedijk, J. Terminology of metal-organic frameworks and coordination polymers (IUPAC Recommendations 2013). Pure Appl. Chem. 2013, 85, 1715-1724.

(2) Slater, A. G., Cooper, A. I. Porous materials. Function-led design of new porous materials. Science 2015, 348, 8075.

(3) Yaghi, O. M.; O'Keeffe, M.; Ockwig, N. W.; Chae, H. K.; Eddaoudi, M., Kim, J. Reticular synthesis and the design of new materials. Nature 2003, 423, 705-714.

(4) Bennett, T. D.; Cheetham, A. K.; Fuchs, A. H., Coudert, F.-X. Interplay between defects, disorder and flexibility in metal-organic frameworks. Nat. Chem. 2016, 9, 11-16.

(5) Chang, Z.; Yang, D.-H.; Xu, J.; Hu, T.-L., Bu, X.-H. Flexible Metal-Organic Frameworks: Recent Advances and Potential Applications. Adv. Mater. 2015, 27, 5432-5441.

(6) Schneemann, A.; Bon, V.; Schwedler, I.; Senkovska, I.; Kaskel, S., Fischer, R. A. Flexible metalorganic frameworks. Chem. Soc. Rev. 2014, 43, 6062-6096.

(7) Horike, S.; Shimomura, S., Kitagawa, S. Soft porous crystals. Nat. Chem. 2009, 1, 695-704.

(8) Fukushima, T.; Horike, S.; Inubushi, Y.; Nakagawa, K.; Kubota, Y.; Takata, M., Kitagawa, S. Solid solutions of soft porous coordination polymers: fine-tuning of gas adsorption properties. Angew. Chem. Int. Ed. 2010, 49, 4820-4824.

(9) Elsaidi, S. K.; Mohamed, M. H.; Banerjee, D., Thallapally, P. K. Flexibility in Metal-Organic Frameworks: A fundamental understanding. Coord. Chem. Rev. 2018, 358, 125-152. 
(10) Coudert, F.-X. Responsive Metal-Organic Frameworks and Framework Materials: Under Pressure, Taking the Heat, in the Spotlight, with Friends. Chem. Mater. 2015, 27, 1905-1916.

(11) Liu, Y.; Her, J.-H.; Dailly, A.; Ramirez-Cuesta, A. J.; Neumann, D. A., Brown, C. M. Reversible structural transition in MIL-53 with large temperature hysteresis. J. Am. Chem. Soc. 2008, 130, 11813-11818.

(12) Rodriguez, J.; Beurroies, I.; Loiseau, T.; Denoyel, R., Llewellyn, P. L. The direct heat measurement of mechanical energy storage metal-organic frameworks. Angew. Chem. Int. Ed. 2015, 54, 46264630.

(13) Lyndon, R.; Konstas, K.; Ladewig, B. P.; Southon, P. D.; Kepert, C. J., Hill, M. R. Dynamic PhotoSwitching in Metal-Organic Frameworks as a Route to Low-Energy Carbon Dioxide Capture and Release. Angew. Chem. 2013, 125, 3783-3786.

(14) Férey, G., Serre, C. Large breathing effects in three-dimensional porous hybrid matter: facts, analyses, rules and consequences. Chem. Soc. Rev. 2009, 38, 1380-1399.

(15) Engel, E. R.; Jouaiti, A.; Bezuidenhout, C. X.; Hosseini, M. W., Barbour, L. J. Activation-Dependent Breathing in a Flexible Metal-Organic Framework and the Effects of Repeated Sorption/Desorption Cycling. Angew. Chem. Int. Ed. 2017, 56, 8874-8878.

(16) Lin, R.-B.; Xiang, S.; Xing, H.; Zhou, W., Chen, B. Exploration of porous metal-organic frameworks for gas separation and purification. Coord. Chem. Rev. 2019, 378, 87-103.

(17) Dong, X.-Y.; Huang, H.-L.; Wang, J.-Y.; Li, H.-Y., Zang, S.-Q. A Flexible Fluorescent SCC-MOF for Switchable Molecule Identification and Temperature Display. Chem. Mater. 2018, 30, 2160-2167.

(18) Vanduyfhuys, L.; Rogge, S. M. J.; Wieme, J.; Vandenbrande, S.; Maurin, G.; Waroquier, M., van Speybroeck, V. Thermodynamic insight into stimuli-responsive behaviour of soft porous crystals. Nat. Commun. 2018, 9, 204.

(19) Yot, P. G.; Boudene, Z.; Macia, J.; Granier, D.; Vanduyfhuys, L.; Verstraelen, T.; van Speybroeck, V.; Devic, T.; Serre, C., Férey, G. et al. Metal-organic frameworks as potential shock absorbers: the case of the highly flexible MIL-53(Al). Chem. Commun. 2014, 50, 9462-9464.

(20) Loiseau, T.; Serre, C.; Huguenard, C.; Fink, G.; Taulelle, F.; Henry, M.; Bataille, T., Férey, G. A rationale for the large breathing of the porous aluminum terephthalate (MIL-53) upon hydration. Chem. Eur. J. 2004, 10, 1373-1382.

(21) Millange, F.; Serre, C., Férey, G. Synthesis, structure determination and properties of MIL-53as and MIL-53ht: the first Criii hybrid inorganic-organic microporous solids: $\mathrm{Cr}$ iii $(\mathrm{OH}) \cdot\left\{\mathrm{O}_{2} \mathrm{C}-\mathrm{C}_{6} \mathrm{H}_{4}-\right.$ $\left.\mathrm{CO}_{2}\right\} \cdot\left\{\mathrm{HO}_{2} \mathrm{C}-\mathrm{C}_{6} \mathrm{H}_{4}-\mathrm{CO}_{2} \mathrm{H}\right\}_{\times}$. Chem. Commun. 2002, 822-823.

(22) Serre, C.; Millange, F.; Thouvenot, C.; Noguès, M.; Marsolier, G.; Louër, D., Férey, G. Very Large Breathing Effect in the First Nanoporous Chromium(III)-Based Solids: MIL-53 or $\mathrm{Cr}^{\prime \prime \prime}(\mathrm{OH}) \cdot\left\{\mathrm{O}_{2} \mathrm{C}-\mathrm{C}_{6} \mathrm{H}_{4}-\mathrm{CO}_{2}\right\} \cdot\left\{\mathrm{HO}_{2} \mathrm{C}-\mathrm{C}_{6} \mathrm{H}_{4}-\mathrm{CO}_{2} \mathrm{H}\right\}_{\times} \cdot \mathrm{H}_{2} \mathrm{O}_{y}$. J. Am. Chem. Soc. 2002, 124, 13519-13526.

(23) Klein, N.; Herzog, C.; Sabo, M.; Senkovska, I.; Getzschmann, J.; Paasch, S.; Lohe, M. R.; Brunner, E., Kaskel, S. Monitoring adsorption-induced switching by (129)Xe NMR spectroscopy in a new metalorganic framework Ni(2)(2,6-ndc)(2)(dabco). Phys. Chem. Chem. Phys. 2010, 12, 11778-11784.

(24) Klein, N.; Hoffmann, H. C.; Cadiau, A.; Getzschmann, J.; Lohe, M. R.; Paasch, S.; Heydenreich, T.; Adil, K.; Senkovska, I., Brunner, E. et al. Structural flexibility and intrinsic dynamics in the M2(2,6ndc)2(dabco) ( $\mathrm{M}=\mathrm{Ni}, \mathrm{Cu}, \mathrm{Co}, \mathrm{Zn}$ ) metal-organic frameworks. J. Mater. Chem. 2012, 22, 10303.

(25) Wang, Z., Cohen, S. M. Modulating metal-organic frameworks to breathe: a postsynthetic covalent modification approach. J. Am. Chem. Soc. 2009, 131, 16675-16677.

(26) Henke, S.; Schneemann, A.; Wütscher, A., Fischer, R. A. Directing the breathing behavior of pillared-layered metal-organic frameworks via a systematic library of functionalized linkers bearing flexible substituents. J. Am. Chem. Soc. 2012, 134, 9464-9474.

(27) Evans, J. D.; Garai, B.; Reinsch, H.; Li, W.; Dissegna, S.; Bon, V.; Senkovska, I.; Fischer, R. A.; Kaskel, S., Janiak, C. et al. Metal-organic frameworks in Germany: From synthesis to function. Coord. Chem. Rev. 2019, 380, 378-418. 
(28) Shivanna, M.; Yang, Q.-Y.; Bajpai, A.; Patyk-Kazmierczak, E., Zaworotko, M. J. A dynamic and multi-responsive porous flexible metal-organic material. Nat. Commun. 2018, 9, 3080.

(29) Mason, J. A.; Oktawiec, J.; Taylor, M. K.; Hudson, M. R.; Rodriguez, J.; Bachman, J. E.; Gonzalez, M. I.; Cervellino, A.; Guagliardi, A., Brown, C. M. et al. Methane storage in flexible metal-organic frameworks with intrinsic thermal management. Nature 2015, 527, 357-361.

(30) Krause, S.; Bon, V.; Senkovska, I.; Stoeck, U.; Wallacher, D.; Többens, D. M.; Zander, S.; Pillai, R. S.; Maurin, G., Coudert, F.-X. et al. A pressure-amplifying framework material with negative gas adsorption transitions. Nature 2016, 532, 348-352.

(31) Sarkisov, L.; Martin, R. L.; Haranczyk, M., Smit, B. On the flexibility of metal-organic frameworks. J. Am. Chem. Soc. 2014, 136, 2228-2231.

(32) Marmier, A., Evans, K. E. Flexibility in MOFs: do scalar and group-theoretical counting rules work? Dalton Trans. 2016, 45, 4360-4369.

(33) Cockayne, E. Thermodynamics of the Flexible Metal-Organic Framework Material MIL-53(Cr) From First Principles. J. Phys. Chem. C 2017, 121, 4312-4317.

(34) Walker, A. M.; Civalleri, B.; Slater, B.; Mellot-Draznieks, C.; Corà, F.; Zicovich-Wilson, C. M.; Román-Pérez, G.; Soler, J. M., Gale, J. D. Flexibility in a metal-organic framework material controlled by weak dispersion forces: the bistability of MIL-53(Al). Angew. Chem. Int. Ed. 2010, 49, 7501-7503.

(35) Rogge, S. M. J.; Waroquier, M., van Speybroeck, V. Reliably Modeling the Mechanical Stability of Rigid and Flexible Metal-Organic Frameworks. Acc. Chem. Res. 2018, 51, 138-148.

(36) Wieme, J.; Lejaeghere, K.; Kresse, G., van Speybroeck, V. Tuning the balance between dispersion and entropy to design temperature-responsive flexible metal-organic frameworks. Nat. Commun.

2018, 9, 4899.

(37) Kuchta, B.; Formalik, F.; Rogacka, J.; Neimark, A. V., Firlej, L. Phonons in deformable microporous crystalline solids. Z. Kristallogr. 2019, $0,3$.

(38) Demuynck, R.; Rogge, S. M. J.; Vanduyfhuys, L.; Wieme, J.; Waroquier, M., van Speybroeck, V. Efficient Construction of Free Energy Profiles of Breathing Metal-Organic Frameworks Using Advanced Molecular Dynamics Simulations. J .Chem. Theory. Comput. 2017, 13, 5861-5873. (39) Butler, K. T.; Walsh, A.; Cheetham, A. K., Kieslich, G. Organised chaos: entropy in hybrid inorganic-organic systems and other materials. Chem. Sci. 2016, 7, 6316-6324.

(40) Formalik, F.; Fischer, M.; Rogacka, J.; Firlej, L., Kuchta, B. Benchmarking of GGA density functionals for modeling structures of nanoporous, rigid and flexible MOFs. J. Chem. Phys. 2018, 149, 64110.

(41) Wei, W.; Li, W.; Butler, K. T.; Feng, G.; Howard, C. J.; Carpenter, M. A.; Lu, P.; Walsh, A., Cheetham, A. K. An Unusual Phase Transition Driven by Vibrational Entropy Changes in a Hybrid Organic-Inorganic Perovskite. Angew. Chem. Int. Ed. 2018, 57, 8932-8936.

(42) Kieslich, G.; Skelton, J. M.; Armstrong, J.; Wu, Y.; Wei, F.; Svane, K. L.; Walsh, A., Butler, K. T. Hydrogen Bonding versus Entropy: Revealing the Underlying Thermodynamics of the Hybrid OrganicInorganic Perovskite [CH3NH3] PbBr3. Chem. Mater. 2018, 30, 8782-8788.

(43) Butler, K. T.; Svane, K.; Kieslich, G.; Cheetham, A. K., Walsh, A. Microscopic origin of entropydriven polymorphism in hybrid organic-inorganic perovskite materials. Phys. Rev. B 2016, 94.

(44) Ryder, M. R.; Civalleri, B.; Bennett, T. D.; Henke, S.; Rudić, S.; Cinque, G.; Fernandez-Alonso, F., Tan, J.-C. Identifying the role of terahertz vibrations in metal-organic frameworks: from gate-opening phenomenon to shear-driven structural destabilization. Phys. Rev. Lett. 2014, 113, 215502.

(45) Ryder, M. R.; van de Voorde, B.; Civalleri, B.; Bennett, T. D.; Mukhopadhyay, S.; Cinque, G.; Fernandez-Alonso, F.; Vos, D. de; Rudić, S., Tan, J.-C. Detecting Molecular Rotational Dynamics Complementing the Low-Frequency Terahertz Vibrations in a Zirconium-Based Metal-Organic Framework. Phys. Rev. Lett. 2017, 118, 255502. 
(46) Tian, Y.-Q.; Zhao, Y.-M.; Chen, Z.-X.; Zhang, G.-N.; Weng, L.-H., Zhao, D.-Y. Design and generation of extended zeolitic metal-organic frameworks (ZMOFs): synthesis and crystal structures of zinc(II) imidazolate polymers with zeolitic topologies. Chem. Eur. J. 2007, 13, 4146-4154.

(47) Park, K. S.; Ni, Z.; Côté, A. P.; Choi, J. Y.; Huang, R.; Uribe-Romo, F. J.; Chae, H. K.; O'Keeffe, M., Yaghi, O. M. Exceptional chemical and thermal stability of zeolitic imidazolate frameworks. Proc. Natl. Acad. Sci. 2006, 103, 10186-10191.

(48) Wharmby, M. T.; Henke, S.; Bennett, T. D.; Bajpe, S. R.; Schwedler, I.; Thompson, S. P.; Gozzo, F.; Simoncic, P.; Mellot-Draznieks, C., Tao, H. et al. Extreme Flexibility in a Zeolitic Imidazolate

Framework: Porous to Dense Phase Transition in Desolvated ZIF-4. Angew. Chem. Int. Ed. 2015, 54, 6447-6451.

(49) Henke, S.; Wharmby, M. T.; Kieslich, G.; Hante, I.; Schneemann, A.; Wu, Y.; Daisenberger, D., Cheetham, A. K. Pore closure in zeolitic imidazolate frameworks under mechanical pressure. Chem. Sci. 2018, 9, 1654-1660.

(50) Vervoorts, P.; Hobday, C. L.; Ehrenreich, M. G.; Daisenberger, D., Kieslich, G. The Zeolitic Imidazolate Framework ZIF-4 under Low Hydrostatic Pressures. Z. Anorg. Allg. Chem. 2019, 645, 970974.

(51) Drużbicki, K.; Pinna, R. S.; Rudić, S.; Jura, M.; Gorini, G., Fernandez-Alonso, F. Unexpected Cation Dynamics in the Low-Temperature Phase of Methylammonium Lead lodide: The Need for Improved Models. J. Phys. Chem. Lett. 2016, 7, 4701-4709.

(52) Mitchell, P. C. H.; Parker, S. F.; Ramirez-Cuesta, A. J., Tomkinson, J. Vibrational Spectroscopy with Neutrons 3; WORLD SCIENTIFIC, 2005.

(53) Togo, A., Tanaka, I. First principles phonon calculations in materials science. Scripta Materialia 2015, 108, 1-5.

(54) Howard, J.; Boland, B. C., Tomkinson, J. Intensities in inelastic neutron scattering spectra: A test of recent theory. Chem. Phys. 1983, 77, 145-151.

(55) Arnold, O.; Bilheux, J. C.; Borreguero, J. M.; Buts, A.; Campbell, S. I.; Chapon, L.; Doucet, M.; Draper, N.; Ferraz Leal, R., Gigg, M. A. et al. Mantid-Data analysis and visualization package for neutron scattering and $\mu$ SR experiments. Nucl. Instrum. Methods Phys. Sect. A 2014, 764, 156-166.

(56) Fjellvåg, $\varnothing$. S.; Armstrong, J.; Vajeeston, P., Sjåstad, A. O. New Insights into Hydride Bonding, Dynamics, and Migration in La2LiHO3 Oxyhydride. J. Phys. Chem. Lett. 2018, 9, 353-358.

(57) Ott, C.; Reiter, F.; Baumgartner, M.; Pielmeier, M.; Vogel, A.; Walke, P.; Burger, S.; Ehrenreich, M.; Kieslich, G., Daisenberger, D. et al. Flexible and Ultrasoft Inorganic 1D Semiconductor and Heterostructure Systems Based on SnIP. Adv. Funct. Mater. 2019, 29, 1900233.

(58) Pinna, R. S.; Rudić, S.; Parker, S. F.; Armstrong, J.; Zanetti, M.; Škoro, G.; Waller, S. P.; Zacek, D.; Smith, C. A., Capstick, M. J. et al. The neutron guide upgrade of the TOSCA spectrometer. Nucl. Instrum. Methods Phys. Sect. A 2018, 896, 68-74.

(59) Grimme, S.; Antony, J.; Ehrlich, S., Krieg, H. A consistent and accurate ab initio parametrization of density functional dispersion correction (DFT-D) for the 94 elements H-Pu. J. Chem. Phys. 2010, $132,154104$.

(60) Hoffman, A. E.J.; Wieme, J.; Rogge, S. M.J.; Vanduyfhuys, L., van Speybroeck, V. The impact of lattice vibrations on the macroscopic breathing behavior of MIL-53(Al). Z. Kristallogr. 2019, 234, 529545.

(61) Fultz, B. Vibrational thermodynamics of materials. Prog. Mater. Sci. 2010, 55, 247-352.

(62) Dove, M. T. Theory of displacive phase transitions in minerals. Am. Mineral. 1997, 82, 213-244.

(63) Tao, J.Z., Sleight, A.W. The role of rigid unit modes in negative thermal expansion. J. Solid State Chem. 2003, 173, 442-448.

(64) Coates, C. S., Goodwin, A. L. How to quantify isotropic negative thermal expansion: magnitude, range, or both? Mater. Horiz. 2019, 3, 831. 
(65) Hu, L.; Chen, J.; Sanson, A.; Wu, H.; Guglieri Rodriguez, C.; Olivi, L.; Ren, Y.; Fan, L.; Deng, J., Xing, $X$. New Insights into the Negative Thermal Expansion: Direct Experimental Evidence for the "GuitarString" Effect in Cubic ScF3. J. Am. Chem. Soc. 2016, 138, 8320-8323.

(66) Mouhat, F., Coudert, F.-X. Necessary and sufficient elastic stability conditions in various crystal systems. Phys. Rev. B 2014, 90.

(67) Muñoz, J. A.; Lucas, M. S.; Delaire, O.; Winterrose, M. L.; Mauger, L.; Li, C. W.; Sheets, A. O.; Stone, M. B.; Abernathy, D. L., Xiao, Y. et al. Positive vibrational entropy of chemical ordering in FeV. Phys. Rev. Lett. 2011, 107, 115501.

(68) Greaves, G. N.; Meneau, F.; Majérus, O.; Jones, D. G., Taylor, J. Identifying vibrations that destabilize crystals and characterize the glassy state. Science 2005, 308, 1299-1302.

(69) Sharp, K. Entropy-enthalpy compensation: fact or artifact? Protein Sci. 2001, 10, 661-667.

(70) Smith, H. L.; Li, C. W.; Hoff, A.; Garrett, G. R.; Kim, D. S.; Yang, F. C.; Lucas, M. S.; Swan-Wood, T.; Lin, J. Y. Y., Stone, M. B. et al. Separating the configurational and vibrational entropy contributions in metallic glasses. Nature Phys 2017, 13, 900-905.

(71) Cheetham, A. K.; Kieslich, G., Yeung, H. H.-M. Thermodynamic and Kinetic Effects in the Crystallization of Metal-Organic Frameworks. Acc. Chem. Res. 2018, 51, 659-667.

(72) Hughes, J. T.; Bennett, T. D.; Cheetham, A. K., Navrotsky, A. Thermochemistry of zeolitic imidazolate frameworks of varying porosity. J. Am. Chem. Soc. 2013, 135, 598-601.

(73) Phillips, A. E. Rigid units revisited. Acta Cryst. A 2018, 74, 406-407.

(74) Schneemann, A.; Rudolf, R.; Baxter, S. J.; Vervoorts, P.; Hante, I.; Khaletskaya, K.; Henke, S.;

Kieslich, G., Fischer, R. A. Flexibility control in alkyl ether-functionalized pillared-layered MOFs by a $\mathrm{Cu} / \mathrm{Zn}$ mixed metal approach. Dalton Trans. 2019, 48, 6564-6570.

(75) Withers, R. L.; Tabira, Y.; Liu, Y., Höche, T. A TEM and RUM study of the inherent displacive flexibility of the fresnoite framework structure type. Phys. Chem. Minerals 2002, 29, 624-632.

(76) Campbell, B.; Howard, C. J.; Averett, T. B.; Whittle, T. A.; Schmid, S.; Machlus, S.; Yost, C., Stokes, H. T. An algebraic approach to cooperative rotations in networks of interconnected rigid units. Acta Cryst. A 2018, 74, 408-424. 
TABLE OF CONTENTS IMAGE

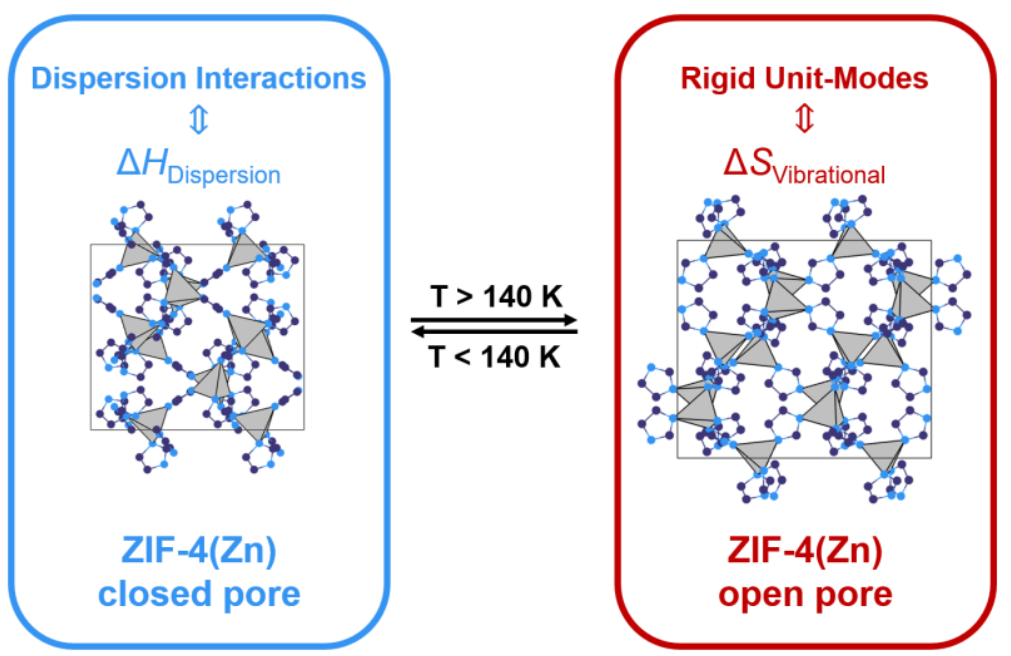

